\title{
LA PRODUCTIVIDAD TOTAL DE FACTORES EN EL SECTOR MANUFACTURERO CHILENO
}

\author{
Claudio Candia Campano* \\ Medardo Aguirre González** \\ Natalia Correa Farias ${ }^{* * *}$ \\ María José Herrera González****
}

T a industria manufacturera ha sido uno de los sectores más imLportantes de la economía chilena. Sin embargo, a pesar de su importancia, desde finales de los años noventa se observa un descenso de su aporte relativo a la producción y al empleo, debido en parte a las pérdidas de productividad que ha enfrentado el sector.

Diversos autores atribuyen esas pérdidas a la baja flexibilidad microeconómica para enfrentar choques negativos que Chile ha experimentado desde $1998^{1}$, lo que se ha traducido en un ajuste insuficiente de los factores productivos en un escenario que requiere otras capacidades. En Chile y en América Latina, la evolución de la productividad se ha examinado principalmente considerando la productividad total de los factores (PTF) y utilizando el método del residuo de Solow. Aquí se utiliza un método distinto, el índice de Malmquist con análisis envolvente de datos (DEA, por su sigla en inglés). Este trabajo analiza la evolución de la PTF en la industria manufacturera chilena durante el periodo de estancamiento de la productividad (1998-2010) y propone una metodología complementaria al análisis clásico.

* Investigador de la Facultad de Economía y Negocios de la Universidad de Talca, Talca, Chile, [clcandia@utalca.cl].

** Doctor de la Universidad de Santiago de Chile y profesor de la Facultad de Economía y Negocios de la Universidad de Talca, Talca, Chile, [maguirre@utalca.cl].

**** Investigadora de la Facultad de Economía y Negocios de la Universidad de Talca, Talca, Chile, [ceoc@utalca.cl].

***** Investigadora de la Facultad de Economía y Negocios de la Universidad de Talca, Talca, Chile, [ceoc@utalca.cl]. Fecha de recepción: 11-03-2015, fecha de modificación: 24-08-2015, fecha de aceptación: 20-10-2016. Sugerencia de citación: Candia C., C.; M. Aguirre, N. Correa y M. J. Herrera, "La productividad total de factores en el sector manufacturero chileno", Revista de Economía Institucional 18, 35, 2016, pp. 229-255. Dor: http://dx.doi.org/10.18601/01245996.v18n35.12.

${ }^{1}$ Ver, p. ej., Beyer y Vergara (2002), Álvarez y Fuentes (2011) y Fuentes (2011). 
En la primera parte se caracteriza la industria manufacturera en general, refiriéndose a su aporte a la economía chilena. En la segunda parte se revisa el análisis habitual de la evolución de la PTF y describe los análisis alternativos derivados de las críticas a ese análisis, como el índice de Malmquist. Después se describen los aspectos metodológicos y se presentan los resultados. Por último se discuten los resultados.

\section{LA INDUSTRIA MANUFACTURERA CHILENA}

La siguiente sección describe la evolución del aporte de la industria manufacturera a la economía chilena y sus principales características.

\section{APORTE DE LA MANUFACTURA A LA ECONOMÍA CHILENA}

E1 sector manufacturero chileno se empezó a desarrollar en 1870. Como fruto de la capitalización de las grandes riquezas mineras y del auge de la agricultura se acumuló un conocimiento que permitió instalar fábricas manufactureras para producir algunos bienes de capital que necesitaban la minería y la agricultura.

Desde entonces, el sector manufacturero creció paulatinamente e hizo un aporte cada vez mayor al рів y al empleo en comparación con otros sectores. Así se convirtió en un sector fundamental que agregaba valor a los insumos y los transformaba en productos finales o intermedios. Hasta comienzos de este siglo su aporte al PIв se mantuvo relativamente estable en torno al $17 \%$, pero desde entonces ha decaído, como se observa en la gráfica 1 , y hoy solo aporta entre un $10 \%$ y un $11 \%$, y un porcentaje muy parecido en términos de empleo, un $12 \%$, que lo convierte en el segundo sector más importante como generador de empleo.

A pesar de la disminución de su aporte a la producción y el empleo, el sector manufacturero sigue siendo muy importante, y hoy es uno de los tres sectores más influyentes en la economía chilena, lo cual suscita interés para investigar las causas de esa disminución.

Si bien el crecimiento de otros sectores es una causa de la disminución del aporte relativo de las manufacturas, también lo es su pérdida de productividad desde comienzos de siglo, la cual coincide con una pérdida de productividad de la economía chilena en su conjunto. Conociendo a priori la disminución de la productividad de la industria manufacturera entre 1998 y 2010, este trabajo somete a prueba la hipótesis general del aumento de su PTF, intentando rechazar esa hipótesis. 
Gráfica 1

Aporte relativo de la industria manufacturera al Рів y al empleo (Porcentaje)

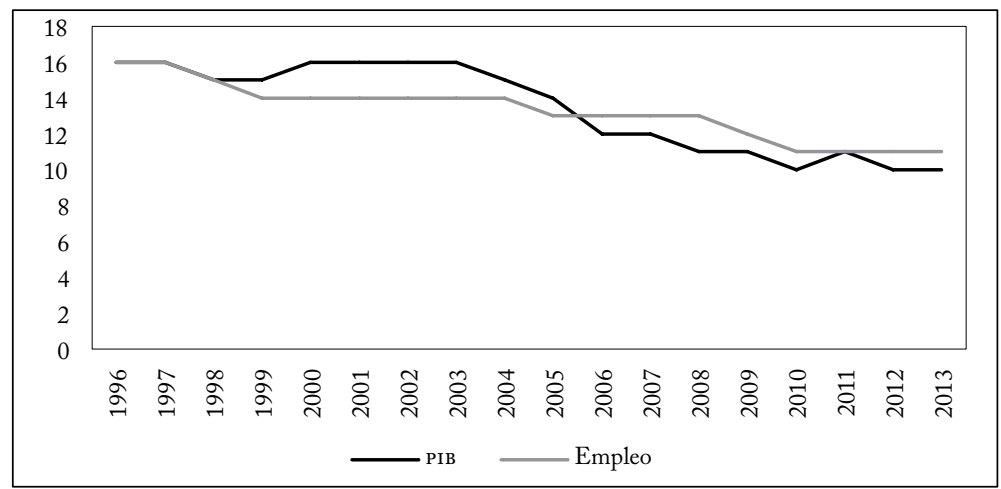

Fuente: elaboración propia con datos del Banco Central de Chile y del ine.

\section{CARACTERÍSTICAS GENERALES DE LA INDUSTRIA}

La siguiente descripción de las características generales de la industria manufacturera chilena se basa principalmente en información obtenida de la Encuesta Nacional Industrial Anual (EnIA), del Banco Central de Chile y de la Sociedad de Fomento Fabril.

Los subsectores más importantes son alimentos, bebidas y tabaco, que representan un 38\% de la producción del sector, seguidos por químicos, petróleo, caucho y plástico, con cerca del 20\%, a los que siguen productos metálicos, maquinarias y equipos, con un $19 \%$.

Gráfica 2

Participación de los factores en el costo de producción de la manufactura (Porcentaje)

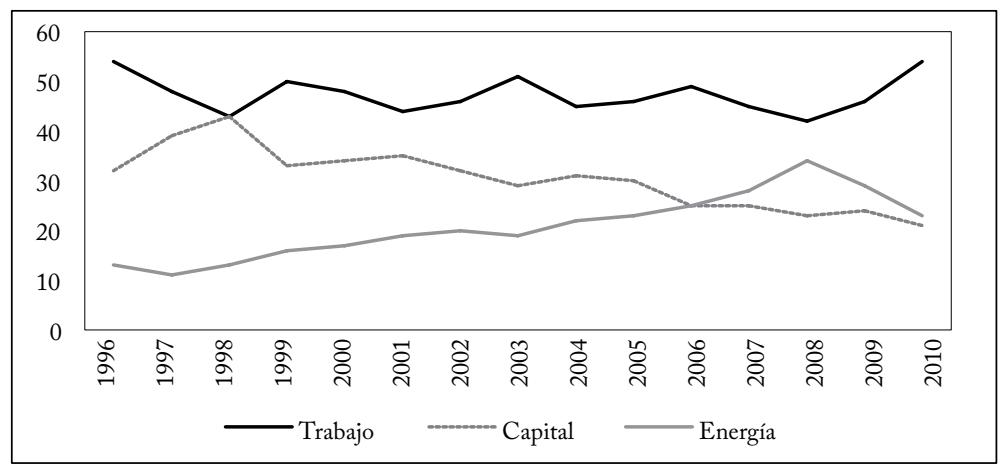

Fuente: ENIA, elaboración propia. 
En materia de propiedad, el número de empresas domésticas (94\%) es mucho mayor que el de las de propiedad extranjera (6\%), y el número de empresas no exportadoras (79\%) es también mayor que el de las exportadoras (21\%), las cuales exportan principalmente salmón (22\%), celulosa (22\%), vinos (13\%), refinados y derivados de petróleo (11\%), a Asia (27\%), el Nafta (19\%), Europa (18\%), la Comunidad Andina (14\%), el Mercosur (12\%), África y Oceanía (6\%) y Centro América y el Caribe (3\%).

En lo que respecta al tamaño, predominan las pequeñas empresas, con menos de 50 trabajadores (67\%), seguidas de las medianas, de 50 a 149 trabajadores (21\%); solo hay un $12 \%$ de empresas grandes, con 150 o más trabajadores. En cuanto al uso de los factores, es mayor la intensidad del trabajo que la del capital, y el ya alto costo de la energía ha aumentado en los últimos años.

\section{REVISIÓN DE LA LITERATURA}

La siguiente sección revisa el papel de la productividad en la teoría del crecimiento económico, los enfoques y el método principal para analizar la productividad. Luego se describe la experiencia de medición de la PTF en Chile y en América Latina, y se exponen los métodos alternativos surgidos de la crítica al método tradicional. Más adelante se muestra cómo ha evolucionado el índice de Malmquist y cómo se estima.

\section{PRODUCTIVIDAD Y TEORÍAS DE CRECIMIENTO ECONÓMICO}

Los avances en el estudio de los cambios de productividad se enmarcan principalmente en la teoría neoclásica del crecimiento, que asocia el crecimiento económico a las mejoras de productividad provenientes del cambio tecnológico y la organización de la producción. Los modelos que se usan suponen rendimientos decrecientes de la inversión en capital físico y humano, y esto implica que los países deben converger hacia niveles de producto y tasas de crecimiento similares. Lo anterior significa un crecimiento acelerado del ingreso y la productividad de los países pobres en relación con los ricos, y por tanto una convergencia de las tasas de crecimiento del ingreso per cápita a lo largo del tiempo. En oposición a la hipótesis de convergencia, la teoría del crecimiento endógeno atribuye un papel protagónico a la acumulación de factores, derivada principalmente de la inversión en capital físico y humano, así como de actividades de investigación y desarrollo. En estos modelos, 
que suponen rendimientos constantes del capital, no se alteran las diferencias de desarrollo entre países pobres y ricos ${ }^{2}$.

\section{ENFOQUES PARA ANALIZAR LA PRODUCTIVIDAD}

En el análisis de la productividad se identifican dos enfoques: el de la productividad parcial (PP) y el de la PTF. El enfoque de la PP compara los cambios en el producto total asociados a cambios de factores productivos específicos, como el capital o el trabajo, considerando así productividades individuales, mientras que el enfoque de la PTF compara los cambios en el producto total asociados a cambios en todos los factores productivos. Dada la insuficiencia de los indicadores parciales de productividad, los economistas se han concentrado en la PTF, entendida desde una perspectiva matemática y desde otra conceptual. Matemáticamente es el cociente entre una función que agrega las salidas y una función que agrega las entradas de los procesos productivos. Conceptualmente, la PTF mide o refleja el progreso tecnológico y las mejoras de eficiencia en los procesos productivos. Prescott (1997), Harberger (1998), Barro (1998) y Helpman (2004) relacionan el crecimiento de la PTF con el avance tecnológico y la eliminación de restricciones, que hace posible que las firmas produzcan más eficientemente.

\section{MEDICIÓN TRADICIONAL DE LA PTF Y SUS CRÍTICAS}

Si bien la literatura difiere en la denominación o nomenclatura de los métodos para analizar la PTF y en su clasificación, hay consenso en lo siguiente. Para estimar la evolución de la PTF se usan dos tipos de métodos, unos basados en la contabilidad del crecimiento y otros en números índice. Una segunda clasificación los divide en paramétricos y no paramétricos, de acuerdo con la necesidad de definir a priori una forma funcional para estimar la PTF: los métodos paramétricos requieren esa forma, los no paramétricos no la requieren. Los métodos basados en la contabilidad del crecimiento se pueden abordar desde ambas perspectivas (paramétrica y no paramétrica) mientras que los basados en números índice son siempre no paramétricos.

Como indica Bernal (2010) luego de revisar la literatura relevante, el método más utilizado es el del residuo de Solow, método pionero en la contabilidad del crecimiento, que se aborda principalmente de

2 Algunos de los trabajos más influyentes sobre las teorías del crecimiento neoclásica y de crecimiento endógeno son Romer (1986), Abramovitz (1986), Baumol (1986), Madisson (1987), Lucas (1988), Barro (1991), Rebelo (1991), Grossman y Helpman (1991), Barro y Sala-i-Martin (1992) y Mankiw et al. (1992). 
manera paramétrica y se describe en Solow (1957): la PTF es una variable no observable en forma directa y se representa mediante la fracción de la producción no explicada por los factores productivos utilizados. Por ello, para calcular la PTF es necesario suponer la función de producción, es decir, una aproximación matemática de cómo se combinan los factores en la producción de bienes y servicios para estimar su residuo, el cual representa la PTF.

Por esta razón, algunos autores dicen que este residuo es "una medida de nuestra ignorancia”, que se ha intentado reducir a medida que pasa el tiempo para entender las causas del crecimiento (Pombo, 1999; Sotelsek y Laborda, 2010). Aunque este método es el más utilizado, no está exento de críticas, las cuales se refieren a los supuestos neoclásicos que adopta el método y a los problemas econométricos asociados a la eficiencia de los estimadores, debido a la existencia de endogeneidad en los modelos. Se han elaborado métodos que responden específicamente a cada tipo de crítica, los cuales se revisan concisamente más adelante.

\section{LA PRODUCTIVIDAD EN CHILE}

Diversos autores resaltan las escasas ganancias de PTF en la economía chilena en forma global y en la mayoría de sus sectores durante los últimos años. Además, observan un punto de inflexión en su tasa de crecimiento antes del año 2000. Según Fuentes et al. (2008), desde 1998, la PTF sufrió un quiebre estructural y dio término a los "años de oro" del crecimiento (1986-1997), con una tasa de crecimiento muy baja desde entonces. En el primer periodo la tasa de crecimiento anual de la PTF era del 3,1\%, mientras que entre 1998 y 2007 fue de un $0,6 \%$.

Según Magendzo y Villena (2012), en 2010 el comité de expertos del Ministerio de Hacienda estimó una tasa de crecimiento promedio anual de la PTF del 3,1\% en el periodo 1991-1997 y del -0,1\% en el periodo 1999-2007. Descenso que habría sido mucho mayor, una tasa inferior al $-2 \%$ en el segundo periodo, si se hubiesen considerado los años de crisis 1998, 2008 y 2009.

Se han hecho otros trabajos sobre la reducción de la tasa de crecimiento de la $\mathrm{PTF}^{3}$. Aunque en esos trabajos se observan diferentes

3 Entre ellos de Gregorio (1997), Rojas et al. (1997), Roldós (1997), Coeymans (1999), Chumacero y Fuentes (2002, 2006), Bergoeing et al. (2002), Beyer y Vergara (2002), Contreras y García (2002), Gallego y Loayza (2002), Álvarez y Fuentes (2004 y 2011), Caballero et al. (2004), Fuentes et al. (2006), Vergara y Rivero (2006), Álvarez et al. (2008), Schwellnus (2010), Fuentes y Morales (2011) y Fuentes (2011). 
matices metodológicos, la base del cálculo de la PTF es el procedimiento de Solow. Magendzo y Villena (2012) así lo corroboran, y señalan que es el método estándar en los países desarrollados y en casi todos los estudios sobre Chile, donde los más importantes suponen una función de producción simple tipo Cobb-Douglas.

\section{LA PRODUCTIVIDAD EN AMÉRICA LATINA}

Igual que en Chile, para estudiar la evolución de la productividad en América Latina se considera la PTF y se usa el método paramétrico del residuo de Solow (Benavente, 2009). El estudio de la PTF hoy ocupa un lugar importante en la investigación económica de la región, pues algunos autores argumentan que es la causa principal del bajo crecimiento en las últimas décadas (Bosworth y Collins, 2003), especialmente durante los años ochenta (Loayza et al. 2004), conocidos como la "década perdida" porque América Latina vivió una recesión y un ajuste macroeconómico con tasas de crecimiento decepcionantes (Martínez, 2008).

Otra causa del interés por la evolución de la PTF es su correlación con otras variables. Un ejemplo es el trabajo de Blyde et al. (2010), que analiza la relación de largo plazo entre fuertes caídas del nivel de producción y la PTF (calculada usando el residuo de Solow y una función Cobb-Douglas) en 71 economías desarrolladas y en desarrollo durante el periodo 1960-2003. Los resultados indican que los colapsos están asociados sistemáticamente a pérdidas o destrucción de PTF de largo plazo, sobre todo en países de África y América Latina, en particular en los años ochenta en esta última región. Señalan además que el país latinoamericano típico experimentó cerca de dos colapsos en esas cuatro décadas.

Según el bid (2010), si bien los países de América Latina y el Caribe registraron avances en algunos indicadores económicos y sociales en el periodo 1994-2008, el crecimiento económico de largo plazo se rezagó con respecto a otras economías emergentes y desarrolladas. No tanto por los impedimentos a la acumulación de factores sino por el déficit crónico de crecimiento de la productividad, caracterizado por una PTF (de nuevo calculada mediante el residuo de Solow) baja y estancada. Según sus estimaciones, la productividad de América Latina apenas llega a la mitad de su potencial y no va a la par de la frontera de productividad, lo que aumenta la brecha de ingresos con el resto del mundo.

Lugones et al. (2007) analizan los avances en materia de cambio tecnológico logrados por un conjunto de países de América Latina y 
el Caribe en 1972-2003, periodo que en sus últimos 15 a 20 años se caracteriza por la expansión del comercio internacional y la atracción de inversión extranjera directa (IED). Como parte del trabajo analizaron la PTF y construyeron un conjunto de indicadores cuantitativos para medir la evolución de la capacidad tecnológica de los distintos países. Sus resultados indican que durante el periodo de estudio, la tasa de crecimiento de la PTF fue relativamente baja con respecto a la economía estadounidense, y muy inferior a la de algunas economías asiáticas de referencia. La tasa de crecimiento promedio de los 18 países latinoamericanos en todo el periodo fue de un - $0,13 \%$.

El trabajo de Coremberg (2012), sobre el periodo subsiguiente, indica que pese al aumento de precios de los productos básicos de exportación de comienzos del siglo, que indujo una visible recuperación económica de América Latina con respecto a la crisis de finales de los noventa, hubo un lento avance de la productividad, a diferencia de lo ocurrido en otras regiones exportadoras de recursos naturales, lo que pone en cuestión la sostenibilidad del crecimiento latinoamericano. Sus resultados muestran que la PTF apenas creció el 0,6\% anual durante la recuperación de 2002-2008, y que si se extiende el análisis hasta 2011, el crecimiento anual de la PTF es nulo, muy inferior al de otras regiones en desarrollo como China e India, el resto de Asia, África y Europa del este, en las que el crecimiento promedio conjunto de la PTF fue del 1,8\% anual. La PTF de la región hacia 2011 era menor que la de 1998 (el nivel máximo alcanzado en los noventa) y en el periodo 2002-2008 solo creció medio punto porcentual por año, impulsada por las mejoras en Perú, Venezuela, Bolivia, Ecuador y Uruguay, compensadas en parte por el menor crecimiento en $\mathrm{Co}^{-}$ lombia y Argentina y la caída en Brasil, Chile y México.

González y Delbianco (2011), que analizan la productividad y algunas variables de la región, intentan medir la correlación entre la apertura al comercio internacional y la PTF de largo plazo (calculada mediante el residuo de Solow) de 20 economías latinoamericanas y caribeñas entre 1960 y 2005. Sus resultados muestran que los choques sobre la PTF no son consecuencia de cambios abruptos en la política comercial (cierre o apertura): los casos de quiebre del nivel de la PTF durante la consolidación de la apertura (1985-1995) no fueron significativos. Esos quiebres coinciden, más bien, con las crisis del petróleo $1973 / 1974$ y 1979/1980 y la crisis mexicana de 1982. Cabe señalar que existe abundante evidencia contraria a estos resultados, tanto en América Latina como en el mundo; por ejemplo, los resultados de Rojas et al. (1997), que indican que el 80\% del crecimiento de la PTF 
en Chile en el periodo 1986-1996 se debe a la mayor participación en el comercio internacional.

Por su parte, Ramírez y Aquino (2005) analizan los efectos de periodos de crisis de inflación (inflación alta y volátil) comprendidos en el periodo 1961-2000 sobre el crecimiento de la PTF para 18 países de América Latina. Sus resultados indican que en la mayoría de estos países la tasa de crecimiento de la PTF tuvo sus mayores caídas durante la "década pérdida", y una relación negativa de largo plazo entre alta inflación y crecimiento de la productividad, así como entre volatilidad de la inflación y crecimiento de la productividad.

Entre los estudios específicos de la industria manufacturera latinoamericana se destaca el trabajo de Ibarrarán et al. (2009). Este trabajo, que usa datos de la Encuesta a Empresas del Banco Mundial (wBEs) de 2006 (y en algunos casos de 2003) para 16 países de América Latina y el Caribe, analiza los determinantes y la magnitud de la brecha de productividad entre empresas grandes y empresas medianas y pequeñas. Además de constatar la brecha de productividad entre empresas de distinto tamaño, sus resultados muestran que las políticas dirigidas a empresas pequeñas y medianas pueden mejorar la PTF, siempre que mejoren los determinantes de las diferencias de productividad con las empresas grandes.

\section{MÉTODOS TRADICIONALES Y ENDOGENEIDAD}

Los modelos tradicionales estiman la PTF como el residuo de la función de producción utilizando el método Mco o técnicas de efectos fijos (EF). Ese procedimiento ha sido criticado porque no da cuenta de la correlación entre factores productivos y productividad, lo que da lugar a un problema de endogeneidad, o existencia de correlación entre el residuo estimado y los factores productivos, el cual a su vez provoca un mayor error en la estimación de los parámetros de los factores y da lugar a una menor confianza en ellos. Por esa razón, en los últimos años se han elaborado métodos para resolver esos problemas, entre los que se destacan los que proponen Olley y Pakes (1996) y Levinsohn y Petrin (2000), quienes para corregir el sesgo usan variables relacionadas con el proceso productivo como proxy de la productividad: la demanda de inversión y la demanda de insumos intermedios.

El procedimiento de Levinsohn y Petrin (2000) es el más utilizado en la literatura, pues como indica Gómez (2011) adopta un marco más general para estimar la productividad y su variable proxy tiene algunas ventajas: el uso de la demanda de insumos intermedios requiere un 
procedimiento más sencillo que el de la demanda de inversión; los insumos intermedios responden suavemente a choques de productividad ya que su ajuste es menos costoso que el de la inversión y, además, porque no toman valores iguales a cero en ningún periodo, como sí puede suceder con la inversión.

E1 procedimiento de Levinsohn y Petrin (2000) parte de una función de producción Cobb-Douglas linealizada logarítmicamente que incluye las variables tradicionales (producto, capital y trabajo) y la variable proxy de productividad. Como no se conoce la forma funcional de la productividad debido a que no es observable, sus coeficientes no se pueden estimar por MCO ni por EF. Recurren entonces a una estimación de dos etapas; en la primera se estiman por Mco los parámetros del trabajo calificado y no calificado, aislando mediante valores esperados la influencia de los demás factores (capital y materias primas), y obtienen estimaciones consistentes para las elasticidades de la mano de obra. En la segunda etapa estiman el coeficiente del capital, asumiendo que la productividad no observable sigue un proceso de Markov de primer orden. Así, el coeficiente del capital se estima con el método generalizado de momentos (MGM), para estimar (como residuo de la función) la PTF mediante Mco, lo que asegura la consistencia de todos los parámetros estimados ${ }^{4}$.

\section{MÉTODOS TRADICIONALES Y SUPUESTOS NEOCLÁSICOS}

La segunda crítica al método tradicional para estimar la PTF apunta a los supuestos neoclásicos en los que se basa: producción en equilibrio, asignación óptima de recursos, ausencia de incertidumbre, rendimientos constantes a escala, mercados perfectos, pleno empleo de los factores y eficiencia de las unidades productivas. Este último supuesto, que considera una igual eficiencia de operación en las unidades de análisis, da lugar a diversas salvedades, entre otras que el crecimiento de la productividad se interpreta como un desplazamiento de la frontera de producción provocado totalmente por un cambio técnico (Dennison, 1974). Es decir, no proporciona información para diferenciar los cambios de la PTF debidos a avances tecnológicos (cambio técnico) de los que resultan de cambios en la eficiencia, y por tanto no permite diferenciar las causas de los cambios de productividad.

Según O’Donnell (2008 y 2010), las limitaciones del método tradicional para estimar la PTF han impulsado el desarrollo de técnicas

${ }^{4}$ En América Latina, algunos trabajos que usan los métodos de Olley y Pakes o Levinsohn y Petrin son Echavarría et al. (2006), Meléndez y Seim (2006) y Gómez (2011) para Colombia; Álvarez y López (2005) para Chile y Tello (2012) para Perú. 
para descomponer la productividad. En vista de ello, en este trabajo no se usan los métodos tradicionales para estimar la PTF y se usa, en cambio, el índice de Malmquist, basado en números índices.

\section{EVOLUCión DEL ÍNDICE DE MALMQUIST}

El índice de Malmquist, que se basa en el cálculo de cocientes de funciones de distancia, permite medir el cambio productivo (crecimiento de la PTF ) que experimenta cualquier unidad que transforme factores productivos así como descomponer ese crecimiento en cambio técnico y cambios de eficiencia técnica.

Los cambios en la eficiencia técnica se entienden como un desplazamiento, en el tiempo, de las unidades de análisis (DMU, por su sigla en inglés) hacia una frontera eficiente de producción (dado que la DMU era ineficiente al inicio del periodo), en tanto que el cambio técnico es el desplazamiento de la frontera debido al progreso tecnológico experimentado por las DMU. Mientras que la mejora de eficiencia, conocida como convergencia a la frontera, refleja mejores prácticas de gestión del proceso productivo, el cambio técnico refleja el conjunto de innovaciones y cambios en las técnicas que desplazan la frontera de producción. Por ello, el índice de Malmquist requiere la estimación previa de fronteras productivas eficientes, las cuales usa como referencia para calcular los cocientes de funciones de distancia.

El índice de Malmquist que aquí se utiliza es el que proponen Färe et al. (1994), el cual es el resultado de una modificación del índice de Malmquist introducido por Caves et al. (1982) y luego mejorado por Färe et al. (1992). Inspirados en el trabajo de Malmquist (1953), Caves et al. emplearon ese índice para medir la variación de la productividad entre dos periodos de tiempo, tomando como medida de los cambios en la productividad total el cociente entre dos funciones de distancia. Según estos autores, dicho índice permite comparar, en dos momentos diferentes, el nivel de insumos, productos y productividad de una misma DMU, o de dos DMU diferentes en el mismo momento o en diferentes momentos. Para analizar los cambios ocurridos entre el periodo inicial y el periodo final se pueden estimar dos índices de productividad, pues cada periodo se puede considerar como periodo de referencia para calcular el índice. Para evitar la arbitrariedad de elegir un periodo $j$ o uno $t$ como referencia, Färe et al. (1992) construyeron un índice de Malmquist que corresponde a la media geométrica de esos dos índices.

Con ese método se puede calcular el índice suponiendo rendimientos constantes a escala, pero no permite descomponer los cambios 
de eficiencia (CE) en cambios en la eficiencia técnica pura (CETP) y cambios en la eficiencia de escala (CEE). Para superar esta dificultad, Färe et al. (1994) propusieron otro método que permite hacer esta diferenciación. Mientras que el cambio en la eficiencia técnica pura refleja el resultado que se obtendría con una gestión puramente técnica de la empresa, sin importar su tamaño, el cambio en la eficiencia de escala se deriva del tamaño de la DMU con respecto a la escala óptima de operación.

Según Färe et al. (1998), Coelli et al. (1998) y Tone (2004), el índice de Malmquist es utilizado en un contexto no paramétrico y el cálculo de las funciones de distancia que requiere se suele realizar con el método DEA.

\section{ESTIMACIÓN DEL ÍNDICE DE MALMQUiST}

El presente análisis exige estimar índices de Malmquist, para lo cual se usa el método propuesto en Färe et al. (1994), mediante el cual el cociente de las distancias de cada combinación de insumos y producto mide el cambio de la PTF entre dos periodos. Es decir, el índice de Malmquist (orientado al producto) representa una función de distancia que estima el máximo cambio proporcional requerido en el nivel de producción para que la combinación de factores y producto en un periodo de referencia sea factible al usar la tecnología de otro periodo.

Así, considerando un nivel de producción $q$, un conjunto de factores productivos $x$ y de tecnologías en los periodos $j \mathrm{y} t$, que se representan respectivamente como:

$d_{0}^{j}\left(q_{j}, x_{j}\right) ; d_{0}{ }^{t}\left(q_{t}, x_{t}\right)$

el índice de Malmquist (orientado al producto) se puede expresar de dos maneras: primera, como muestra la ecuación (2), utilizando como referencia la tecnología del periodo $j$, y segunda, como muestra la ecuación (3), utilizando como referencia la tecnología del periodo $t$.

$m_{0}^{j}\left(q_{j}, x_{j}, q_{t}, x_{t}\right)=\frac{d_{0}^{j}\left(q_{t}, x_{t}\right)}{d_{0}^{j}\left(q_{j}, x_{j}\right)}$

$m_{0}{ }^{t}\left(q_{j}, x_{j}, q_{t}, x_{t}\right)=\frac{d_{0}^{t}\left(q_{t}, x_{t}\right)}{d_{0}^{t}\left(q_{j}, x_{j}\right)}$

Además de las tecnologías de referencias, la estimación de los índices de Malmquist requiere calcular la distancia de la observación del periodo $t$ con respecto a la tecnología del periodo $j$, y la distancia de 
la observación del periodo $j$ con respecto a la tecnología del periodo $t$, las cuales son, respectivamente:

$d_{0}^{j}\left(q_{t}, x_{t}\right) ; d_{0}^{t}\left(q_{j}, x_{j}\right)$

Un valor de $m_{\circ}$ mayor que 1 indica un crecimiento de la PTF entre un periodo y otro, y un valor menor que 1 indica una disminución.

Para evitar la elección arbitraria de la tecnología de referencia se suele definir el índice de Malmquist como la media geométrica de estos dos índices (Fisher, 1992; Caves et al., 1982), es decir:

$m_{0}\left(q_{j}, x_{j}, q_{t}, x_{t}\right)=\left[\frac{d_{0}^{j}\left(q_{t}, x_{t}\right)}{d_{0}^{t}\left(q_{j}, x_{j}\right)} \cdot \frac{d_{0}^{t}\left(q_{t}, x_{t}\right)}{d_{0}^{t}\left(q_{j}, x_{j}\right)}\right]^{1 / 2}$

Reorganizando las funciones de distancia se obtiene una nueva expresión que permite diferenciar el índice de cambio de la eficiencia técnica y el índice de cambio técnico:

$m_{0}\left(q_{j}, x_{j}, q_{t}, x_{t}\right)=\frac{d_{0}^{t}\left(q_{t}, x_{t}\right)}{d_{0}^{j}\left(q_{j}, x_{j}\right)}\left[\frac{d_{0}^{j}\left(q_{t}, x_{i}\right)}{d_{0}^{t}\left(q_{t}, x_{t}\right)} \cdot \frac{d_{0}^{j}\left(q_{j}, x_{j}\right)}{d_{0}{ }^{t}\left(q_{j}, x_{j}\right)}\right]^{1 / 2}$

En la expresión anterior el componente situado fuera de los corchetes mide el cambio de la eficiencia técnica (orientado al producto) entre j y $t$. La parte restante mide el cambio técnico o media geométrica del cambio en la tecnología. Además, como ya se observó, Färe et al. (1994) sugieren dividir el cambio de la eficiencia técnica en eficiencia de escala y eficiencia técnica pura, lo que implica descomponer el cambio de eficiencia del primer término en esos dos componentes, como se observa en las ecuaciones (7) y (8), correspondientes al cambio en la eficiencia pura y al cambio en la eficiencia de escala, respectivamente:

$\frac{d_{0 v}^{t}\left(q_{t}, x_{t}\right)}{d_{0 v}^{t}\left(q_{j}, x_{j}\right)}$

$\left[\frac{d^{t}{ }_{0 v}\left(q_{t}, x_{t}\right) / d^{t}{ }_{0 c}\left(q_{t}, x_{t}\right)}{d^{t}\left(q_{t}, x_{t}\right) / d^{t}\left(q_{0 c}, x_{j}\right)} \cdot \frac{d^{j}{ }_{0 v}\left(q_{t}, x_{t}\right) / d^{j}{ }_{0 c}\left(q_{t}, x_{t}\right)}{d^{j}{ }_{0 v}\left(q_{t}, x_{t}\right) / d^{j}{ }_{0 c}\left(q_{j}, x_{j}\right)}\right]^{1 / 2}$

El cambio en la eficiencia de escala de la ecuación (8) es la media geométrica de dos medidas de cambio de la eficiencia de escala: una con respecto a la tecnología del periodo $t$ y otra con respecto a la tecnología del periodo $j$. 


\section{Programación lineal E ÍNDICE DE MALMQUIST}

Para calcular el índice se requiere estimar las cuatro medidas de distancia de la ecuación (5), lo cual exige plantear los problemas de programación lineal respectivos. Suponiendo rendimientos constantes, se debe optimizar la función objetivo, sometida a las siguientes restricciones (Färe et al., 1994):

$\left[d_{0}^{\prime}\left(q_{j}, x_{j}\right)\right]^{-1}=\max _{0, \alpha}$

$-\phi q_{i j}+Q_{j} \lambda \geq 0 ; x_{i j}-X \lambda \geq 0 ; \lambda \geq 0$

$\left[d_{0}^{\prime}\left(q_{i}, x_{t}\right)\right]^{-1}=\max , \alpha \phi$

$-\phi q_{i}+Q, \lambda \geq 0 ; x_{i t}-X, \lambda \geq 0 ; \lambda \geq 0$

$\left[d_{0}^{\prime}\left(q_{t}, x_{t}\right)\right]^{-1}=\max , ., \phi$

$-\phi q_{i t}+Q, \lambda \geq 0 ; x_{i n}-X, \lambda \geq 0 ; \lambda \geq 0$

$\left[d_{0}^{\prime}\left(q_{j}, x_{j}\right)\right]^{-1}=\max _{0}, \lambda \phi$

$-\phi q_{i j}+Q \lambda \geq 0 ; x_{i j}-X, \lambda \geq 0 ; \lambda \geq 0$

Para descomponer el cambio de la eficiencia técnica en sus dos componentes, los problemas de programación lineal (9), (10), (11) y (12) se deben modificar incorporando la restricción de convexidad que materializa el supuesto de rendimientos variables a escala, quedando definidos en forma general como sigue:

$\left[d_{0}\left(q_{,}, x_{0}\right)\right]^{-1}=\max _{0, \lambda} \phi$

$-\phi q_{i v}+Q, \lambda \geq 0 ; x_{i}-X, \lambda \geq 0 ; \lambda \geq 0 ; e^{T} \lambda=1$

donde $e$ es un vector $\mathrm{N} \times 1$ c, y $\mathrm{N}$ es el número de DMU.

La restricción incorporada exige que haya convexidad, de modo que la frontera de eficiencia productiva debe estar conformada por segmentos que unan sus puntos extremos; así, por el momento solo se obtienen medidas de eficiencia técnica pura (sin medidas de eficiencia de escala). Por ello no se puede saber si una DMU opera con rendimientos crecientes o decrecientes a escala. Para resolver este problema se debe remplazar la restricción de convexidad por otra que impida que las DMU operen con rendimientos crecientes. Así, la expresión general anterior se transforma en:

$\left[d_{0}^{*}\left(q_{,}, x_{0}\right)\right]^{-1}=\max _{\phi}, \phi \phi$

$-\phi q_{i v}+Q, \lambda \geq 0 ; x_{i,}-X, \lambda \geq 0 ; \lambda \geq 0 ; e^{T} \lambda \leq 1$

El carácter de las eficiencias de escala de una DMU específica se determina comparando las medidas de eficiencia técnica obtenida me- 
diante (13), que solo permite rendimientos variables a escala, y (14), que solo permite rendimientos constantes o decrecientes. Si el tipo de rendimientos coincide, la DMU tiene rendimientos decrecientes, de lo contrario se asumen rendimientos crecientes.

Así, con base en las funciones de distancia que caracterizan la tecnología de acuerdo con la mejor práctica se calcula el índice de Malmquist que descompone el cambio en la PTF en cambio en la eficiencia técnica pura, cambio en la eficiencia de escala y cambio tecnológico.

\section{METODOLOGÍA}

En esta sección se describen las fuentes de datos, el método de análisis, los componentes de la función objetivo que se busca optimizar y el tratamiento de los datos para obtener los paneles respectivos.

Las fuentes de datos secundarios corresponden a tres paneles construidos a partir de la ENIA que realiza el Instituto Nacional de Estadística (INE). Se construyen paneles para cada tipo de DMU (divisiones, grupos y clases), conformados a partir de la información sobre los establecimientos manufactureros de diez o más trabajadores durante el periodo 1998-2010, tomando en cuenta la clasificación industrial internacional uniforme (CIIU), donde una división reúne un conjunto de grupos, y estos reúnen un conjunto de clases.

\section{MÉTODO DE ANÁLISIS}

Como ya se indicó, las funciones de distancia para calcular el índice de Malmquist se estiman a partir de fronteras productivas técnicamente eficientes, las cuales se calculan con el método DEA. Estas fronteras eficientes son el punto de referencia para evaluar las variaciones en el desempeño productivo de los tipos de DMU, calcular el índice de Malmquist y estimar las variaciones de la PTF y sus componentes.

El método DEA, propuesto por Charnes et al. (1978), consiste en construir una frontera tecnológica con las unidades productivas que usan las mejores prácticas, escogiendo una orientación específica, hacia los insumos o hacia el producto. La frontera se construye punto a punto, donde cada uno representa el máximo nivel de producto alcanzable con cierta combinación de insumos, o el mínimo nivel de insumos necesario para cierto nivel de producto. Así, los puntos de la frontera representan combinaciones eficientes de factores productivos y nivel de producción. En este trabajo se utiliza una función de distancia orientada al producto y se propone maximizar la producción 
de un vector de insumos, lo que refleja la orientación productiva de la industria manufacturera chilena.

E1 DEA tiene varias ventajas: 1 ) no exige una función matemática que relacione insumos y productos, lo que evita eventuales sesgos de especificación; 2) caracteriza cada unidad mediante un puntaje único de eficiencia; 3) permite usar diferentes unidades de medida para evaluar la eficiencia; 4) incluye en forma simultánea insumos y productos; 5) define el conjunto de unidades que se pueden utilizar como referencia para mejorar la productividad y permite encontrar los valores óptimos de insumos y productos para que cada unidad sea eficiente (Coll y Blasco, 2006). Entre sus principales problemas cabe mencionar que no supone ningún tipo de forma funcional, lo que impide diferenciar cambios de productividad a causa de ineficiencias y a causa de factores aleatorios (perturbaciones de los modelos paramétricos), y la significancia estadística mediante pruebas de hipótesis de los coeficientes de los factores productivos (ibíd.).

Se utilizó el Data Envelopment Analysis Program (DEAP) que permite calcular el índice de Malmquist propuesto por Färe et al.

\section{LA FUNCIÓN DE PRODUCCIÓN}

La función de producción propuesta, que relaciona los factores productivos utilizados en la industria manufacturera chilena y el nivel de producción que contribuyen a alcanzar, es la siguiente.

$Q_{i t}=f\left(L_{t}, K_{t}, E_{t}, I_{t}\right)$

Cuadro 1

Componentes de la función de producción de la manufactura chilena

\begin{tabular}{lcc}
\hline Símbolo & Nombre & Estimadores \\
\hline Oit & Producción & Valor agregado neto \\
Lit & Rerabajo & Remuneración pagada \\
Kit & Capido activo fijo terrenos \\
& & Saldo activo fijo edificios \\
& Saldo activo fijo maquinaria \\
& Saldo activo fijo vehículos \\
Eit & Valor neto electricidad comprada \\
& Valor neto de combustibles \\
Iit & Materiales de reparación \\
& Mnergía & Material de oficina \\
& Materias primas traspasadas \\
& Insumos & Materias primas importadas \\
& & Artículos comprados para reventa \\
\hline
\end{tabular}

Todas las variables se miden en miles de pesos de 2009.

Fuente: ENIA, elaboración propia. 
E1 cuadro 1 describe las variables incluidas en la función de producción señalando el símbolo de los componentes, su nombre y las variables empleadas como estimadores. Cada símbolo indica que se tiene información del componente respectivo para la DMU $i$ durante el año $t$. Es necesario aclarar, además, que el valor de un componente en cada DMU y cada año es el resultado de la suma de sus estimadores.

\section{TRATAMIENTO DE LOS DATOS}

Para construir un panel de datos que permitiera determinar la frontera eficiente mediante el DEA se resolvieron dos problemas. El primero, la falta de información o de información adecuada (insumos o productos con valores negativos) de ciertas DMU en algunos años del periodo. E1 segundo, la conversión de las variables en unidades monetarias corrientes a términos reales (miles de pesos de un año base).

Para resolverlos se identificaron y seleccionaron únicamente las divisiones, grupos y clases de las que se disponía de información para todos los años del periodo 1998-2010; así se obtuvieron tres paneles (uno para cada tipo de unidad de corte transversal o DMU). Luego se eliminaron las unidades de corte transversal que durante algún periodo presentaran un valor agregado neto negativo, porque el DEA no funciona con valores negativos de los insumos o productos.

Si bien los paneles construidos son balanceados a nivel de las unidades de corte indicadas (divisiones, grupos y clases), no lo son a nivel de empresas, porque cada año se remplazan empresas que forman parte de la industria y, por tanto, de la muestra consultada.

E1 cuadro 2 muestra las DMU de cada tipo de panel. E1 primero tiene 17 divisiones, el segundo 37 grupos y el tercero 71 clases. Para estas DMU hay información adecuada de todo el periodo. Para obtener valores comparables en cada panel se identifica el único deflactor disponible para todo el periodo, el IPC, que se usa para expresar los valores en miles de pesos de 2009. El cuadro 3 muestra el nivel de representatividad promedio anual en todo el periodo para cada panel. La representatividad promedio anual, de acuerdo con la proporción del valor agregado neto, es del 91,2\% para clases, del 93,4\% para grupos y del 95,3\% para divisiones. El cuadro 4 muestra los valores del IPC. 
Cuadro 2

DMU utilizadas según codificación cııU Rev. 3

\begin{tabular}{lccccccccc}
\hline Divisiones & 15 & 17 & 18 & 19 & 20 & 21 & 22 & 24 & 25 \\
& 26 & 27 & 29 & 31 & 33 & 34 & 35 & 36 & \\
\hline Grupos & 151 & 152 & 154 & 155 & 171 & 172 & 173 & 181 & 191 \\
& 192 & 201 & 202 & 210 & 221 & 222 & 241 & 242 & 251 \\
& 252 & 261 & 269 & 271 & 272 & 291 & 292 & 293 & 311 \\
& 312 & 315 & 319 & 332 & 341 & 342 & 343 & 351 & 361 \\
\hline Clases & 159 & & & & & & & & \\
& 1552 & 1512 & 1513 & 1514 & 1520 & 1531 & 1541 & 1543 & 1549 \\
& 1912 & 1920 & 2010 & 2021 & 2022 & 2023 & 2029 & 2101 & 2102 \\
& 2109 & 2211 & 2219 & 2221 & 2222 & 2411 & 2412 & 2413 & 2421 \\
& 2422 & 2423 & 2424 & 2511 & 2519 & 2520 & 2610 & 2692 & 2694 \\
& 2695 & 2696 & 2699 & 2710 & 2720 & 2913 & 2915 & 2919 & 2921 \\
& 2924 & 2925 & 2929 & 2930 & 3120 & 3150 & 3190 & 3311 & 3320 \\
\hline
\end{tabular}

Fuente: ENIA, elaboración propia.

Cuadro 3

Representatividad anual de las unidades de corte, 1998-2010 (Porcentaje)

\begin{tabular}{cccc}
\hline $\begin{array}{c}\text { Divisiones } \\
\text { cIIU Rev. 3 }\end{array}$ & $\begin{array}{c}\text { Promedio anual } \\
\text { de clases (\%) }\end{array}$ & $\begin{array}{c}\text { Promedio anual } \\
\text { de grupos (\%) }\end{array}$ & $\begin{array}{c}\text { Promedio anual } \\
\text { de divisiones (\%) }\end{array}$ \\
\hline 15 & 94,0 & 93,4 & 100,0 \\
17 & 91,5 & 98,1 & 100,0 \\
18 & 100,0 & 100,0 & 100,0 \\
19 & 100,0 & 100,0 & 100,0 \\
20 & 99,1 & 100,0 & 100,0 \\
21 & 99,6 & 100,0 & 100,0 \\
22 & 79,5 & 98,5 & 100,0 \\
24 & 93,0 & 99,4 & 100,0 \\
25 & 100,0 & 100,0 & 100,0 \\
26 & 91,6 & 99,9 & 100,0 \\
27 & 99,9 & 99,9 & 100,0 \\
28 & 0,0 & 0,0 & 0,0 \\
29 & 74,1 & 98,2 & 100,0 \\
30 & 0,0 & 0,0 & 0,0 \\
31 & 63,4 & 93,4 & 100,0 \\
32 & 0,0 & 0,0 & 0,0 \\
33 & 48,2 & 29,3 & 100,0 \\
34 & 98,7 & 98,7 & 100,0 \\
35 & 75,2 & 75,0 & 100,0 \\
36 & 98,3 & 99,9 & 100,0 \\
$99 / D$ & 0,0 & 0,0 & 0,0 \\
Total & 91,24 & 93,37 & 95,29 \\
\hline
\end{tabular}

Fuente: ENIA, elaboración propia. 
Cuadro 4

Cambio histórico del IPC

$(2009=100)$

\begin{tabular}{cccccc}
\hline Año & IPC & Año & IPC & Año & IPC \\
\hline 1995 & 57,49 & 2001 & 73,88 & 2007 & 90,64 \\
1996 & 61,72 & 2002 & 78,42 & 2008 & 98,54 \\
1997 & 68,85 & 2003 & 80,63 & 2009 & 100,00 \\
1998 & 71,15 & 2004 & 81,48 & 2010 & 101,41 \\
1999 & 65,50 & 2005 & 83,96 & & \\
2000 & 76,52 & 2006 & 86,81 & & \\
\hline
\end{tabular}

Fuente: INE (2013), elaboración propia.

\section{ÍNDICE DE MALMQUIST PROMEDIO PONDERADO}

E1 DEAP calcula un índice de Malmquist para cada año y cada DMU. Por ello, para obtener el índice de Malmquist anual para la industria manufacturera se deben promediar los índices calculados de todas las DMU involucradas en ese año. El promedio puede ser simple o ponderado. Aquí se estimó un promedio ponderado considerando la importancia relativa de cada DMU en el valor agregado neto de la industria en dicho año. Cabe aclarar que como resultado se obtiene un índice de Malmquist ponderado para cada año del periodo para los tres paneles, y que cada índice se calcula con ponderaciones cambiantes porque el peso relativo de las DMU en términos de valor agregado neto no es constante en el tiempo. Las gráficas 3, 4 y 5 muestran la evolución de la PTF y de sus componentes (eficiencia técnica y cambio técnico) de acuerdo con el índice de Malmquist ponderado.

\section{RESULTADOS}

Los resultados indican que la disminución de la PTF durante el periodo de estudio se debe al cambio técnico y no a la eficiencia técnica, la cual permanece constante, es decir que no ha habido cambios en la eficiencia pura ni en la de escala.

La gráfica 3 muestra la evolución del índice de Malmquist para las divisiones de la industria manufacturera. Los resultados indican que la PTF se redujo un $11,9 \%$ promedio anual durante el periodo y que se empezó a desacelerar a finales de 1998-2010. La causa de esa reducción fue el cambio técnico y no la eficiencia técnica. Mientras que esta permanece constante, el cambio técnico, igual que la PTF, presenta una disminución desacelerada.

Los resultados del índice de Malmquist y de sus componentes para los grupos y clases de la industria manufacturera son muy similares, es decir, también indican una reducción desacelerada de la PTF estimada, a un $11,9 \%$ anual, a causa de la disminución del cambio 
técnico y no de la eficiencia en el uso de los recursos disponibles. La gráfica 4 muestra la evolución de la PTF y de sus componentes considerando los grupos de la industria manufacturera como unidad de corte transversal.

Gráfica 3

Cambio en la PTF y sus componentes, divisiones

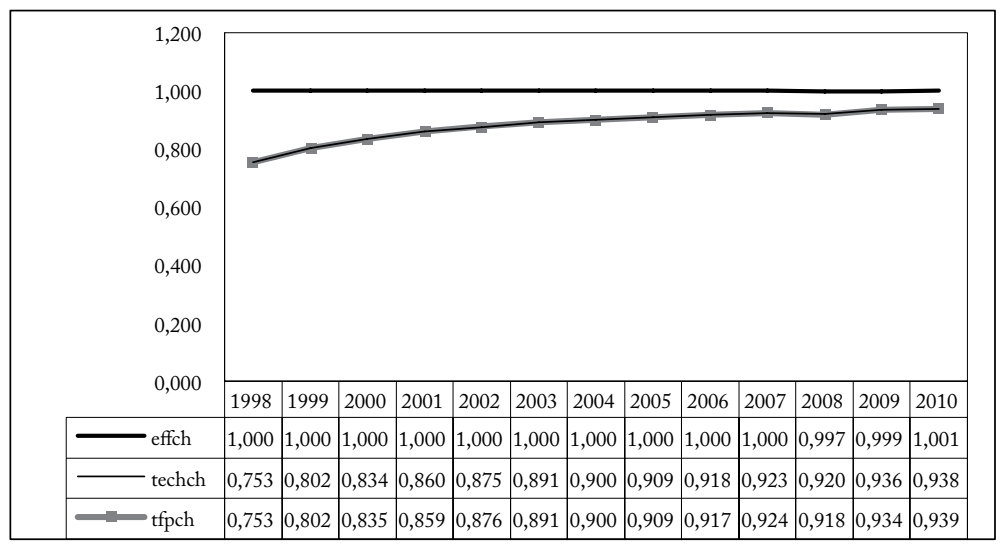

Fuente: elaboración propia.

Gráfica 4

Cambio en la PTF y sus componentes, grupos

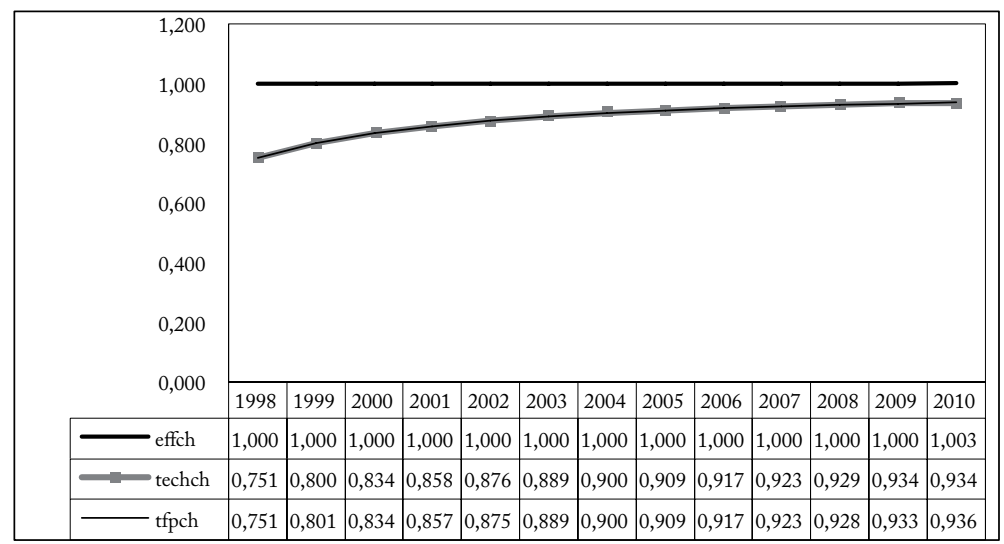

Fuente: elaboración propia.

Por su parte, la gráfica 5 muestra la evolución de la PTF y de sus componentes considerando las clases de la industria manufacturera como unidad de corte transversal. 
Gráfica 5

Cambio en la PTF y sus componentes, clases

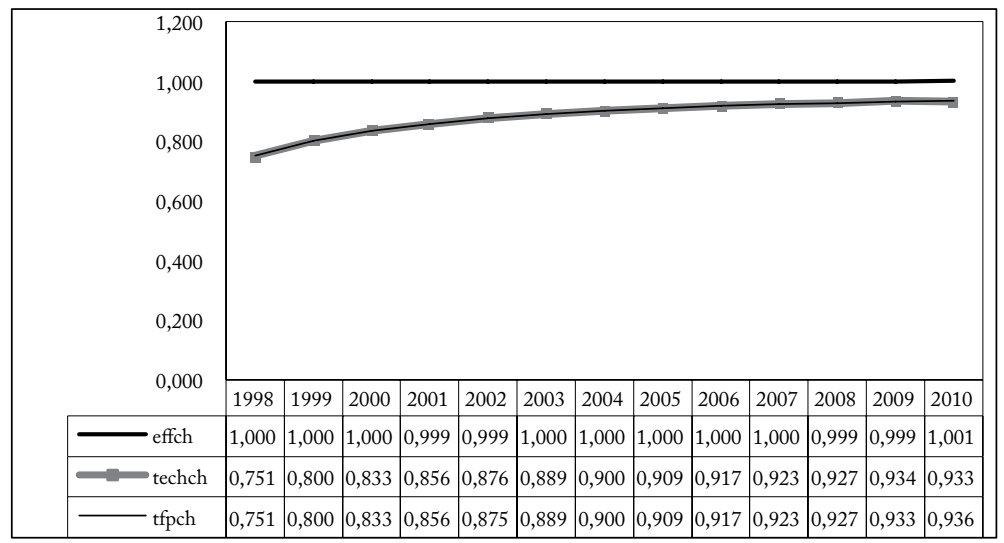

Fuente: elaboración propia.

\section{DISCUSIÓN}

Como ya se mencionó, diversos trabajos intentan explicar la evolución de la PTF de la economía chilena, cuya conclusión general es que las políticas macroeconómicas y las reformas microeconómicas contribuyeron al alto crecimiento de la PTF agregada en los "años dorados" de la economía chilena.

En cuanto a las causas de la reducción de la tasa del incremento de la PTF durante 1998 y 2010 , esos trabajos las agrupan de diferentes maneras y subrayan distintos aspectos. Por ejemplo, Magendzo y Villena (2012) las dividen en factores externos y exógenos a la economía chilena, y en factores internos y de carácter más permanente asociados a la falta de políticas públicas adecuadas. En el primer grupo incluyen el encarecimiento de la energía, la crisis asiática, el boom del cobre asociado a la tasa de cambio real y los rendimientos marginales de la PTF. En el segundo grupo incluyen la inflexibilidad microeconómica, la escasez de capital humano adecuado, el encarecimiento de los costos logísticos, la falta de competencia, la escasez de capital social, la deficiente inversión en investigación y desarrollo y la volatilidad de la tasa de cambio.

Por su parte, Fuentes (2011) considera que la reducción de la tasa de crecimiento de la PTF agregada se debe principalmente a la baja flexibilidad microeconómica para enfrentar choques negativos. Desde finales de los años noventa, ante choques negativos y debido a restricciones para ajustar los factores productivos, la economía chilena no se ha ajustado de manera óptima, lo que ha elevado los costos de 
producción. Esta baja flexibilidad se manifiesta en las altas restricciones y los costos para ajustar eficientemente el trabajo, mientras que los choques negativos han sido generados por el aumento del salario mínimo y la crisis asiática (1998) y por el aumento de precios de la energía (2004) ${ }^{5}$. En cuanto a la PTF de la manufactura, Vergara y Rivero (2006), Álvarez y Fuentes (2011) y Magendzo y Villena (2012) encuentran que ha seguido un patrón similar al de la PTF agregada y que ha sido afectada por los mismos factores que han desacelerado su tasa de crecimiento desde comienzos de siglo.

La comparación de los resultados de esos trabajos (que usan métodos paramétricos) con los de este estudio (que usa un método no paramétrico) muestra similitudes y diferencias. Una similitud es que se encuentran tasas anuales promedio de crecimiento negativas (aunque de distinta magnitud) en los periodos respectivos. Una diferencia es la evolución de la PTF. Esos trabajos encuentran cambios drásticos en la tasa de crecimiento entre un año y otro (de negativo a positivo y viceversa), mientras que aquí se encuentra un cambio más estable, negativo y decreciente en todos los años. El trabajo de Magendzo y Villena (2012), el único que analiza un periodo similar y comparable (1992-2010), encuentra tasas de crecimiento altamente negativas, de $-15 \%,-9 \%$ y $-7 \%$ en algunos años, seguidas de tasas positivas.

Un punto importante de discusión es la importancia relativa de la energía en la industria manufacturera y su posible efecto en la disminución de la PTF del sector. Álvarez et al. (2008), Echavarría et al. (2008) y Blümel et al. (2010) analizan la relación entre las variaciones de la PTF y los cambios de precios de la energía en Chile. Álvarez et al. usan datos del sector industrial a nivel de planta para analizar la relación entre ese precio de la energía y la productividad laboral en la manufactura chilena, y estiman que el choque energético de los primeros años de la década de 2000 (debido al aumento del precio del petróleo y a la disminución del agua para generar energía eléctrica) explica entre el $20 \%$ y el $60 \%$ de la reducción de su productividad en el periodo 2000-2005, una pérdida del 0,5\% de PTF en esos años a nivel agregado. Encuentran, además, que los sectores más intensivos en energía han experimentado la mayor reducción de la tasa de crecimiento de la productividad.

Cabe señalar, por último, que si bien en Chile se ha usado el índice de Malmquist para estimar el cambio de la PTF en sectores como la educación, la banca, la agricultura y la salud pública, no parece haberse

\footnotetext{
${ }^{5}$ Los resultados de Magendzo y Villena (2012) y Fuentes (2011) son confirmados por Caballero et al. (2004), Beyer y Vergara (2002), y Álvarez y Fuentes (2011).
} 
usado recientemente en el caso de la industria manufacturera, lo que impide cotejar los resultados obtenidos con distintos métodos. En otros países latinoamericanos existen aplicaciones recientes del índice de Malmquist para analizar la evolución de la PTF; por ejemplo, Brown y Domínguez (2013) y Becerril et al. (2013) en México.

\section{CONCLUSIONES}

Este trabajo analiza la evolución de la PTF de la industria manufacturera chilena estimando el índice de Malmquist mediante el método DEA, para así incorporar (con más fuerza) el uso de este índice a la discusión y crítica metodológica relativa a la PTF.

Los resultados del periodo 1998-2010 indican que la PTF disminuyó significativamente durante el periodo, de modo que se rechaza la hipótesis de partida, y que esa reducción se debe ante todo al cambio tecnológico negativo mientras que la eficiencia técnica permanece constante. El rechazo de la hipótesis de trabajo fortalece el uso del método no paramétrico para el análisis de la PTF y, dadas las diferencias con los resultados de los métodos paramétricos, permite hacer comparaciones y discutir la bondad de los métodos.

Una limitación del estudio es que el remplazo habitual de empresas manufactureras impide construir paneles balanceados a ese nivel. Además, no existe un deflactor más adecuado (como el índice de precios al productor, IPP) para todo el periodo, pues aunque el INE publica un IPP para toda la economía y uno para la manufactura, solo están disponibles desde 2003 y 2009 respectivamente.

\section{REFERENCIAS BIBLIOGRÁFICAS}

1. Abramovitz, M. "Catching up, forging ahead and falling behind", Journal of Economic History 46 2, 1986, pp. 385-406.

2. Álvarez, R. y R. Fuentes. Patrones de especialización y crecimiento sectorial en Chile, Banco Central de Chile, working paper 288, 2004.

3. Álvarez, R. y R. Fuentes. Labor market regulations and productivity. Evidence from Chilean manufacturing plants, Instituto de Economía, Pontificia Universidad Católica de Chile, documento de trabajo 396, 2011.

4. Álvarez, R.; A. García, y P. García. Choques de energía y productividad en la industria manufactuera chilena, Banco Central de Chile, working paper 482, 2008.

5. Álvarez, R. y R. A. López. "Exporting and performance: Evidence from Chilean plants", Canadian Journal of Economics 38, 4, 2005, pp. 1384-1400.

6. BID. La era de la productividad: cómo transformar las economías desde sus cimientos, C. Pagés, ed., Nueva York, Pórtico Bookstore, 2010. 
7. Barro, R. J. Notes on growth accounting, NBER, working paper 6654, 1998.

8. Barro, R. J. y X. Sala-i-Martin. "Convergence", Journal of Political Economy 100, 2, 1992, pp. 223-251.

9. Barro, R. J. "Economic growth in a cross section of countries", Quarterly Journal of Economics 106, 2, 1991, pp. 407-443.

10. Baumol, W. J. "Productivity growth, convergence and welfare: What the long-run data show", American Economic Review 76, 5, 1986, pp. 1072-1085.

11. Becerril, O.; O. Rodríguez y T. Chávez. "Productividad multifactorial de los subsectores de la industria manufacturera mexicana”, Explanans 2, 2, 2013, pp. 47-72.

12. Benavente, J. M. El desafio de la innovación para la América Latina de hoy, Cieplan, Serie de estudios socio económicos 52, 2009.

13. Bergoeing, R. et al. "Policy-driven productivity in Chile and Mexico in the 1980s and 1990s", American Economic Review, Papers and Proceedings 92, 2, 2002, pp. 16-21.

14. Beyer, H. y R. Vergara. Productivity and economic growth: The case of Chile, Banco Central de Chile, working paper 174, 2002.

15. Blümel, G.; M. Domper y R. Espinoza. Crecimiento económico, precios de la energía e innovación tecnológica, Libertad y Desarrollo, Serie informe económico 206, 2010.

16. Blyde, J.; Ch. Daude y E. Fernández. "Output collapses and productivity destruction", Review of World Economics 146 2, 2010, pp. 359-387.

17. Bosworth, B. y S. Collins. The empirics of growth: An update, Brookings Institution, Brookings Papers on Economic Activity, Economic Studies Program 34, 2003.

18. Brown, F. y L. Domínguez. "La productividad, reto de la industria mexicana", Comercio Exterior 63, 3, 2013, pp. 12-23.

19. Caballero, R.; E. Engel y A. Micco. "Flexibilidad microeconómica en América Latina”, Revista Economía Chilena 7, 2, 2004, pp. 5-26.

20. Caves, D.; L. Christensen y W. E. Diewert. "The economic theory of index numbers and the measurement of input, output and productivity", Econometrica 50, 6, 1982, pp. 1393-1414.

21. Charnes, A.; W. W. Cooper y E. Rhodes. "Measuring the efficiency of decision making units", European Journal of Operations Research 2, 6, 1978, pp. 429-444.

22. Chumacero, R. y R. Fuentes. On the determinants of the Chilean economic growth, Banco Central de Chile, working paper 134, 2002.

23. Chumacero, R. y R. Fuentes. "Chilean growth dynamics”, Economic Modelling 23, 2, 2006, pp. 197-214.

24. Coelli, T.; D. Prasada-R. y G. E Battesse. An introduction to efficiency and productivity analysis, Boston, Kluwer Academic Publishers, 1998.

25. Coeymans, J. E. "Determinantes de la productividad en Chile", Cuadernos de Economía 36, 107, 1999, pp. 597-637.

26. Coll, V. y O. Blasco. Evaluación de la eficiencia mediante el análisis envolvente de datos, Valencia, Universidad de Valencia-Eumed, 2006.

27. Contreras, G. y P. García. "Estimating gaps and trends for the Chilean economy”, Revista de Economía Chilena 5, 2, 2002, pp. 37-55. 
28. Coremberg, A. "La productividad de América Latina ante el auge de precios de productos básicos", Cuadernos Económicos de ICE 84, 2012, pp. 123-153.

29. De Gregorio, J. "Crecimiento potencial en Chile: una síntesis", F. Morandé y R. Vergara, eds., Análisis empirico del crecimiento económico en Chile, Santiago, Centro de Estudios Públicos, ilades, 1997.

30. Dennison, E. Accounting for United States economic growth 1929-1969, Washington DC, Brookings Institution, 1974.

31. Echavarría, J. J.; M. A. Arbeláez y M. F. Rosales. "La productividad y sus determinantes: el caso de la industria colombiana", Desarrollo $y$ Sociedad 57, 1, 2006, pp. 77-122.

32. Echavarría, G.; P. Jervis y C. Soto. Impacto del costo de la energía en la medición del PIB potencial en el escenario central de proyecciones, Banco Central de Chile, documento preliminar, 2008.

33. Färe, R. et al. "Productivity change in Swedish pharmacies 19801989: A nonparametric Malmquist approach”, Journal of Productivity Analysis 3, 1992, pp. 85-102.

34. Färe, R. et al. "Productivity growth, technical progress, and efficiency change in industrialized countries", American Economic Review 84, 1, 1994, pp. 66-83.

35. Färe, R.; S. Grosskopf y P. Roos. "Malmquist productivity indexes: A survey of theory and practice”, S. Färe y R. Grosskopf, eds., Index numbers: Essays in honour of Sten Malmquist, Boston, Kluwer Academic, 1998.

36. Fisher, I. The making of index numbers, Boston, Houghton Mifflin, 1992.

37. Fuentes, R. Una mirada desagregada del deterioro de la productividad en Chile: ¿̇existe un cambio estructural?, Instituto de Economía, Pontificia Universidad Católica de Chile, documento de trabajo 401, 2011.

38. Fuentes, R.; F. Gredic y M. Larraín. "La brecha del producto en Chile: medición y evaluación”, Economía Chilena 11, 2, 2008, pp. 7-30.

39. Fuentes, R.; M. Larraín y K. Schmidt-H. "Measuring and explaining total factor productivity in Chile”, Cuadernos de Economía 43, 2006, pp. 113-142.

40. Fuentes, R. y M. Morales. "On the measurement of TfP: A latent variable approach", Macroeconomic Dynamics 15, 2, 2011, pp. 145-159.

41. Gallego, F. y N. Loayza. "The golden period for growth in Chile, explanations and forecasts”, N. Loayza y R. Soto, eds., Economic growth: Sources, trends and cycles, Santiago, Banco Central de Chile, 2002.

42. Gómez, A. "Productividad multifactorial del sector manufacturero del Cauca 1993-2006”, Sociedad y Economía 20, 2011, pp. 243-265.

43. González, G. H. y F. A. Delbianco. "Apertura y productividad total de factores: análisis de la contemporaneidad en los quiebres estructurales para América Latina y el Caribe”, Revista de Análisis Económico 26 1, 2011, pp. 53-81.

44. Grossman, G. y E. Helpman. "Quality ladders in the theory of growth", Review of Economic Studies 58, 1991, pp. 43-61.

45. Harberger, A. C. "A vision of the growth process", American Economic Review 88, 1, 1998, pp. 1-32. 
46. Helpman, E. El misterio del crecimiento económico, Barcelona, Antoni Bosch, 2004.

47. Ibarrarán, P.; A Maffioli y R. Stucchi. SME policy and firms'productivity in Latin America, Bonn, Institute for the Study of Labor (IZA), discussion paper 4486, 2009.

48. Levinsohn, J. y A. Petrin. When industries become more productive, do firms?: Investigating productivity dynamics, NBER, working paper 6893, 2000.

49. Loayza, N.; P. Fajnzylber y C. Calderón. Economic growth in Latin American and the Caribbean, Banco Central de Chile, documentos de trabajo 265, 2004.

50. Lucas, R. E. "On the mechanics of economic development", Journal of Monetary Economics 22, 1988, pp. 3-22.

51. Lugones, G. E.; P. Gutti y N. Le Clech. Indicadores de capacidades tecnológicas en América Latina, Cepal México, Serie estudios y perspectivas 89, 2007.

52. Maddison, A. "Growth and slowdown in advanced capitalist economies: Techniques of quantitative assessment", Journal of Economic Literature 25, 2, 1987, pp. 649-698.

53. Magendzo, I. y M. Villena. Evolución de la productividad total de factores, Universidad Adolfo Ibáñez, informe técnico, 2012.

54. Malmquist, S. "Index numbers and indifference curves", Trabajos de Estadistica 4, 1, 1953, pp. 209-242.

55. Mankiw, N. G.; D. Romer y D. N. Weil. "A contribution to the empirics of economic growth", Quarterly Journal of Economics 107, 2, 1992, pp. 407-437.

56. Martínez, A. “Colombia y Venezuela: desempeño económico, tipo de cambio y relaciones Estado-empresarios", Revista de Economía Institucional 10, 19, 2008, pp. 265-291.

57. Meléndez, M. y K. Seim. "La productividad del sector manufacturero colombiano y el impacto de la política comercial: 1977-2001”, Desarrollo y Sociedad 57, 2006, pp. 1- 41.

58. O’Donnell, C. "An aggregate quantity-price framework for measuring and decomposing productivity and profitability change", Centre for Efficiency and Productivity Analysis, University of Queensland, working paper WP072008, 2008.

59. O’Donnell, C. "Measuring and decomposing agricultural productivity and profitability change", Australian Journal of Agricultural and Resource Economics 54, 4, 2010, pp. 527-560.

60. Olley, S. y A. Pakes. "The dynamics of productivity in the telecommunications equipment industry", Econometrica 64, 6, 1996, pp. 1263-1298.

61. Pombo, C. "Productividad industrial en Colombia: una aplicación de números índices", Revista de Economía de la Universidad del Rosario 2, 1, 1999, pp. 107-139.

62. Prescott, E. Needed: A theory of total factor productivity, Federal Reserve Bank of Minneapolis, staff report 242, 1997. 
63. Ramírez, N. y J. C. Aquino. Crisis de inflación y productividad total de los factores en Latinoamérica, Banco Central de Reserva del Perú, documento de trabajo 2005-005, 2005.

64. Rebelo, S. "Long run policy analysis and long run growth", Journal of Political Economy 99, 1991, pp. 500-521.

65. Bernal, J. R. "E1 residuo de Solow revisado", Revista de Economía Institucional 12, 23, 2010, pp. 347-361.

66. Rojas, P.; E. López y S. Jiménez. "Determinantes del crecimiento y estimación del producto potencial en Chile: el rol del comercio internacional", F. Morandé y R. Vergara, eds., Análisis empírico del crecimiento económico en Chile, Santiago, Centro de Estudios Públicos, ILADES, 1997.

67. Roldós, J. "E1 crecimiento del producto potencial en mercados emergentes: el caso de Chile", F. Morandé y R. Vergara, eds., Análisis empírico del crecimiento económico en Chile, Santiago, Centro de Estudios Públicos, ilades, 1997.

68. Romer, P. “Increasing returns and long-run growth", Journal of Political Economy 98, 5, 1986, pp. 71-102.

69. Schwellnus, C. Chile: Boosting productivity growth by strengthening competition, entrepreneurship and innovation, oEcD Economics Department, working paper 785, 2010.

70. Solow, R. "Technical change and the aggregate production function", Review of Economics and Statistics 39, 3, 1957, pp. 312-320.

71. Sotelsek, D. y L. Laborda. "América Latina: medición de la eficiencia productiva y el cambio técnico incorporando factores ambientales”, Revista de la Cepal 101, 2010, pp. 17- 37.

72. Tello, M. "Productividad total factorial en el sector manufacturero del Perú: 2002-2007”, Economía 35, 70, 2012, pp. 103-141.

73. Tone, K. "Malmquist Productivity Index: Efficiency change over time", W. Cooper, L. Seifor y J. Zhu, eds., Handbook on data envelopment analysis, Boston, Kluwer Academic, 2004.

74. Vergara, R. y R. Rivero. "Productividad sectorial en Chile: 1986-2001", Cuadernos de Economía 43, 127, 2006, pp. 143-168. 